\title{
BMJ Open Atrial fibrillation and mortality in outpatients with heart failure in Tanzania: a prospective cohort study
}

\author{
Yunchan Chen (10 , ${ }^{1,2}$ Emmanuel Alphonce, ${ }^{2}$ Eva Mujuni, ${ }^{2}$ Godfrey A Kisigo, ${ }^{1,2}$ \\ Justin R Kingery, ${ }^{1,2}$ Abel Makubi, ${ }^{3}$ RN Peck, ${ }^{1,2}$ Fredrick Kalokola ${ }^{2}$
}

To cite: Chen Y, Alphonce E, Mujuni $\mathrm{E}$, et al. Atrial fibrillation and mortality in outpatients with heart failure in Tanzania: a prospective cohort study. BMJ Open 2022;12:e058200. doi:10.1136/ bmjopen-2021-058200

- Prepublication history for this paper is available online. To view these files, please visit the journal online (http://dx.doi org/10.1136/bmjopen-2021058200).

YC and EA contributed equally.

Received 09 0ctober 2021 Accepted 05 January 2022

Check for updates

(C) Author(s) (or their employer(s)) 2022. Re-use permitted under CC BY-NC. No commercial re-use. See rights and permissions. Published by BMJ.

${ }^{1}$ Weill Cornell Medicine, New York, New York, USA

${ }^{2}$ Department of Internal Medicine, Catholic University of Health And Allied Sciences Weill Bugando School of Medicine, Mwanza, Mwanza, Tanzania, United Republic of

${ }^{3}$ Ministry of Health Community Development Gender Elderly and Children, Dar es Salaam, Dar es Salaam, Tanzania, United Republic of

Correspondence to Dr Fredrick Kalokola; kalokola85@gmail.com

\section{ABSTRACT}

Objective In recent years, the prevalence and mortality of heart failure (HF) and other associated cardiovascular diseases have doubled in sub-Saharan Africa (SSA). Studies in high-income countries indicate that HF with concurrent atrial fibrillation (AF) is linked to increased mortality. Our objective was to determine the incidence and clinical outcomes of $\mathrm{AF}$ among patients with $\mathrm{HF}$ in SSA.

Design A prospective cohort study using data collected between 0ctober 2018 and May 2020.

Setting Outpatient clinic at a tertiary hospital in Mwanza, Tanzania.

Participants 303 adult participants (aged $\geq 18$ years) with HF as defined by the European Society of Cardiology guidelines (2016) and 100 adults with HF as defined by clinical criteria alone were enrolled into the study. Patients with comorbid medical condition that had prognosis of $<3$ months (ie, advance solid tumours, advance haematological malignancies) were excluded. Methods Participants were screened for AF, and their medical history, physical examinations and sociodemographic information were obtained. Multivariable logistic regression models were used to examine factors associated with AF incidence. Cox regression models were used to analyse 3-month mortality and its associated risk factors.

Results We enrolled 403 participants with HF (mean age $60 \pm 19$ years, 234 (58\%) female). The AF prevalence was $17 \%$. In multivariable models, factors associated with AF were low income, alcohol consumption and longer duration of HF. At the end of the 3-month follow-up, 120 out of 403 (30\%) participants died, including $44 \%$ (31/70) of those with AF. Higher heart rate on ECG, more severe New York Heart Association HF class, rural residence and anaemia were significantly correlated with mortality.

Conclusion $\mathrm{AF}$ is common, underdiagnosed and is associated with significant mortality among outpatients with HF in Tanzania (HR 1.749, 95\% Cl 1.162 to 2.633 , $\mathrm{p}=0.007$ ). Our findings additionally identify tachycardia (>110bpm, HR 1.879, 95\% Cl 1.508 to $2.340, \mathrm{p}<0.001$ ) as an easily measurable, high-impact physical examination finding for adverse outcomes in patients with HF.

\section{INTRODUCTION}

As global life expectancy increases, the incidence of heart failure (HF) has risen

\section{Strengths and limitations of this study}

This study is one of the few to examine the prevalence and mortality of atrial fibrillation (AF) among outpatients with heart failure (HF) in sub-Saharan Africa.

- This study focuses on readily accessible physical examination measures, demographics, socioeconomic and lifestyle attributes. They are inexpensive to acquire and are well adapted for risk stratification in resource-limited settings.

- As a cohort study, no causal relationships can be established between the risk factors and mortality. Questionnaire data on social and personal history are contingent on patient report accuracy. Given the limitations in medical equipment, concurrent coronary artery disease, the order in which $\mathrm{AF}$ and $\mathrm{HF}$ developed, and whether HF was due to non-ischaemic or ischaemic causes were not established.

- All participants were recruited from a single healthcare facility, which may qualify the generalisability of the findings.

substantially. ${ }^{1}$ Approximately 26 million people live with HF worldwide, ${ }^{2}$ with lowincome and middle-income countries bearing the greatest burden. ${ }^{13}$ From 1990 to 2013, cardiovascular disease-related deaths in Africa increased twofold, and accounted for roughly $38 \%$ of all non-communicable disease mortalities. ${ }^{1}{ }^{4}$ Within sub-Saharan Africa (SSA), previous studies have indicated an 'epidemiological transition,' whereby chronic, non-communicable diseases are gradually overtaking infectious diseases in prevalence. ${ }^{13}$ In particular, HF constitutes roughly $9.4 \%-42.5 \%$ of all hospital admissions and $25.6 \%-30.0 \%$ of the cardiology clinic visits at institutions across Africa. ${ }^{5} \mathrm{HF}$ has a higher 1-year post-hospital discharge mortality than all other diagnoses. ${ }^{6}$ In addition to patient-level burden, HF poses significant economic strain secondary to recurrent hospitalisations, lost productivity and pharmacological costs. ${ }^{57}$ 
Atrial fibrillation (AF) incidence is also escalating rapidly among new cardiovascular diagnoses. ${ }^{8}$ Between 1990 and 2010, the annual deaths caused by AF grew by 2 -fold and 1.9-fold in men and women, respectively. ${ }^{9}$ While $\mathrm{AF}$ and $\mathrm{HF}$ are known to share common cardiometabolic risk factors, growing evidence suggests that the presence of one may precipitate the severity of the other. Compared with sinus-rhythm, comorbid AF is associated with higher all-cause mortality and hospitalisation rates in patients with HF. ${ }^{10}$ Furthermore, AF-related atrial remodelling, altered ventricular haemodynamics and arrhythmia-induced myopathy are linked to further HF progression. ${ }^{11}$

Despite the synergistic comorbidity of $\mathrm{AF}$ and $\mathrm{HF}$, little is known about the prevalence of $\mathrm{AF}$ among outpatients with HF within SSA, or its impact on clinical outcomes. Therefore, we conducted a prospective cohort study to elucidate the prevalence, correlates and mortality associated with this patient population in Tanzania.

\section{METHODS}

\section{Overview}

This clinic-based prospective cohort study involved 403 patients who were enrolled in a registry of HF. This registry was created as part of a more extensive hospital quality improvement programme for patients with HF. Data collection and follow-up spanned from October 2018 to May 2020.

\section{Setting and participants}

The study was conducted at the outpatient clinic of Bugando Medical Center (BMC), a zonal hospital for the Lake Victoria Zone in northwest Tanzania. BMC serves a population of over 14 million with a 950-bed capacity. In each month, BMC provides care for approximately 400 patients with $\mathrm{HF}$, with an average of 100 patients seen weekly. BMC is similar to other facilities that provide care for HF in Tanzania and Uganda. ${ }^{12} 13$

All patients attending the outpatient clinic with a diagnosis of HF were screened between October and December of 2019. Patients $\geq 18$ years of age and seeking HF care were recruited serially until the target sample size was attained $(\mathrm{n} \geq 331)$. Patients with comorbid medical conditions with a prognosis of $<3$ months (ie, advanced malignancy) were excluded from the study. Of the 403 enrolled patients, 303 had the diagnosis of HF objectively confirmed according to the European Society of Cardiology (ESC) 2016 guidelines,${ }^{14}$ where 133 had heart failure with reduced ejection fraction (HFrEF) and 170 had heart failure with preserved ejection fraction (HFpEF). For the remaining 100 patients, the diagnosis of HF was made according to the Framingham criteria, and in the absence of another primary diagnosis responsible for volume overload. ${ }^{15}$

\section{Study procedures}

Consented participants were interviewed using a standard questionnaire that collected clinical and demographic information such as age, sex, residence, duration of $\mathrm{HF}$ and New York Heart Association (NYHA) functional classification. Participants were also evaluated for palpitations, shortness of breath, syncope or presyncope, exercise intolerance, chest pain and fatigue. Physical examination was performed on every participant. Blood pressure measurements were taken from the right arm using an automated blood pressure monitor after subjects had rested for at least $5 \mathrm{~min}$. Pulse rate was determined, and noted for irregularity, regularity and amplitude, then compared with the heart rate for pulse deficit.

Height was measured using a rigid ruler attached to a wall and rounded to the nearest $0.5 \mathrm{~cm}$. Weight was measured without shoes, with patients wearing light clothing and recorded to the nearest $500 \mathrm{~g}$ using the DETECTO scale. Body mass index (BMI) was calculated using the Quetelet equation ${ }^{16}$ and categorised using the WHO Classification Scale, with underweight BMI classified as $<18.5 \mathrm{~kg} /$ $\mathrm{m}^{2}$, normal BMI as $18.5-24.9 \mathrm{~kg} / \mathrm{m}^{2}$, overweight BMI as $25-29.9 \mathrm{~kg} / \mathrm{m}^{2}$ and obese BMI as $\geq 30 \mathrm{~kg} / \mathrm{m}^{2}$. Additionally, electronic medical records were reviewed to extract blood haemoglobin and serum creatinine values.

Study participants were then subjected to a resting 12-lead electrocardiography. The heart rate on ECG was recorded for all subjects. Tracings with irregular QRS complexes and absent discrete $\mathrm{P}$ waves were categorised as AF, in accordance with the ESC 2016 criteria. ${ }^{17}$ All diagnoses of atrial fibrillation were confirmed by a staff cardiologist. Patients with AF had their results communicated to the attending physician and were treated according to protocol.

\section{Follow-up and outcome determination}

At least three contact phone numbers were obtained at the time of enrolment, including one from the patient and two from friends and relatives. All participants were followed for a period of 3 months, with none lost to follow-up. The research team interviewed the participants during their regularly scheduled visits on a monthly basis. Phone calls were made to those not presenting to clinic. During these interviews, information about their recent medical updates or hospitalisations was collected. If the participant could not be reached, the designated alternate contact was called to determine the patient's vital status. Mortality was ascertained via phone call to each individual family. The families confirmed the death was cardiac in origin or related to their cardiac diagnosis (HF/cardioembolic stroke/cardiorenal syndrome). Additionally, for those who died during hospitalisation, care was taken to confirm with the family member that the original admission was due to cardiac aetiologies.

\section{Statistical analysis}

By the difference in proportions calculation, a minimum sample size of 331 patients was needed to provide at least $80 \%$ power to detect the difference in mortality rates between patients with AF and those without (two-sided test with a $5 \%$ level of significance).$^{18}$ Our pretest estimation 


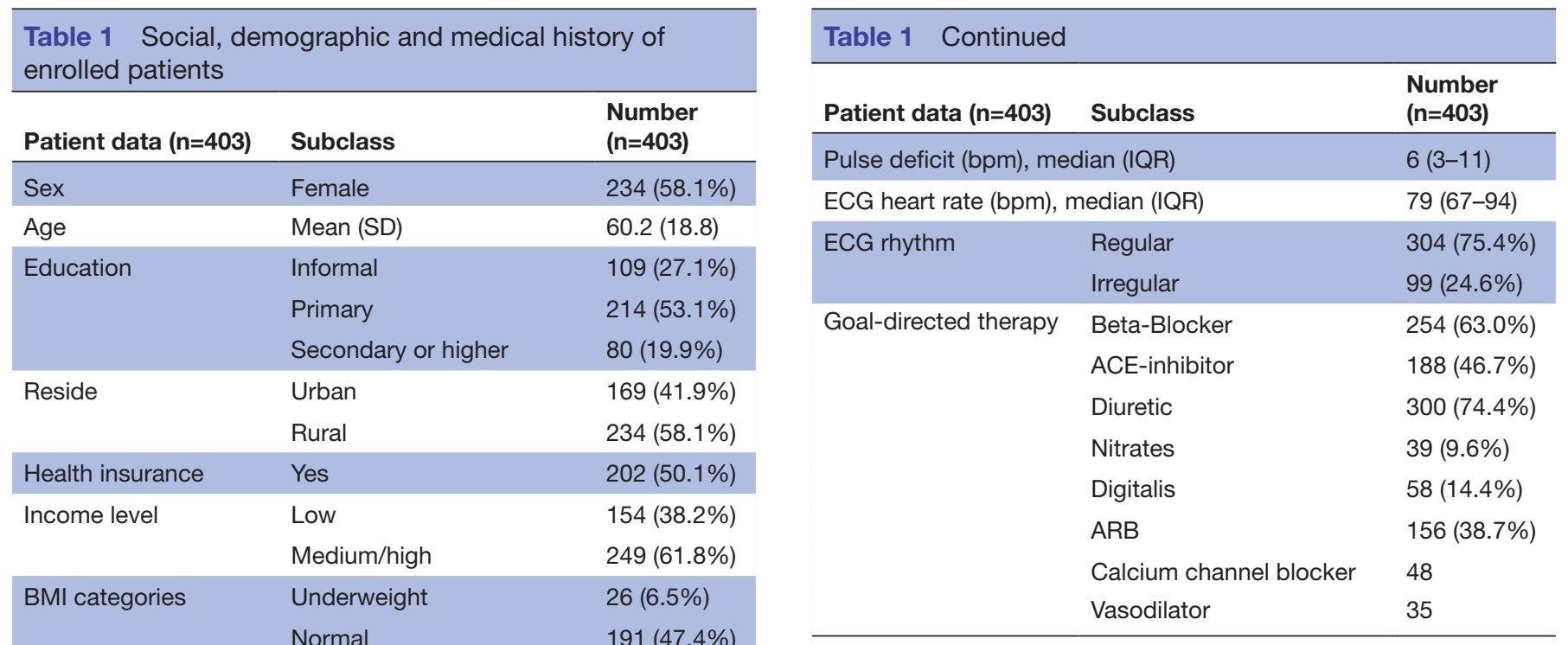

$\begin{array}{ll} & \text { Normal } \\ & \text { Overweight } \\ & \text { Obese/severely obese }\end{array}$

History of hypertension Yes

$191(47.4 \%)$

$120(29.8 \%)$

$66(16.4 \%)$

$323(80.2 \%)$

Duration of heart failure (years), median (IQR) 4 (3-9)

Family history of heart Yes

$180(44.7 \%)$

failure

\begin{tabular}{|c|c|c|}
\hline \multirow[t]{3}{*}{ NYHA function class } & $\|$ & $83(20.6 \%)$ \\
\hline & III & $317(78.7 \%)$ \\
\hline & IV & $3(0.7 \%)$ \\
\hline Diabetes mellitus & Yes & $97(24.1 \%)$ \\
\hline HIV & Positive & $21(5.2 \%)$ \\
\hline Atrial fibrillation & Present (ECG confirmed AF) & $70(17.4 \%)$ \\
\hline \multirow{3}{*}{$\begin{array}{l}\text { Alcohol } \\
\text { (average units of } \\
\text { alcohol/day) }\end{array}$} & Yes & $189(46.9 \%)$ \\
\hline & Median (IQR) & $0(0-10)$ \\
\hline & Range & $0-60$ \\
\hline Cigarette smoking & Yes & 77 (19.1\%) \\
\hline \multirow[t]{3}{*}{ Echo LV EF (\%) } & $<40$ & $133(33.0 \%)$ \\
\hline & $>=40$ & $170(42.2 \%)$ \\
\hline & Unknown & $100(24.8 \%)$ \\
\hline \multirow[t]{3}{*}{ Haemoglobin } & Normal (>12g/dL) & $175(43.4 \%)$ \\
\hline & Mild anaemia (100-119g/L) & $188(46.7 \%)$ \\
\hline & $\begin{array}{l}\text { Moderate/severe anaemia } \\
(<=99 \mathrm{~g} / \mathrm{L})\end{array}$ & $38(9.9 \%)$ \\
\hline \multirow{4}{*}{$\begin{array}{l}\text { Rheumatic heart } \\
\text { disease }\end{array}$} & Positive history and AF & $6(1.5 \%)$ \\
\hline & Positive history and no AF & $18(4.5 \%)$ \\
\hline & Negative history and AF & $64(15.8 \%)$ \\
\hline & Negative history and no AF & $315(78.2 \%)$ \\
\hline \multicolumn{2}{|c|}{ Creatinine level, median (IQR) } & $94(77-169)$ \\
\hline \multicolumn{2}{|c|}{ Systolic blood pressure, median (IQR) } & $\begin{array}{l}122(106- \\
142)\end{array}$ \\
\hline \multicolumn{2}{|c|}{ Diastolic blood pressure, median (IQR) } & $70(66-82)$ \\
\hline \multirow[t]{2}{*}{ Pulse rhythm } & Regular & $300(74.4 \%)$ \\
\hline & $\begin{array}{l}\text { Irregular (diagnosed by } \\
\text { clinical exam) }\end{array}$ & $103(25.6 \%)$ \\
\hline \multicolumn{2}{|c|}{ Heart rate (bpm), median (IQR) } & $79(71-91)$ \\
\hline
\end{tabular}

Continued 
Table 2 Univariate logistic regression for sociodemographic, clinical history and anthropomorphic correlates associated with atrial fibrillation

\begin{tabular}{|c|c|c|c|c|c|}
\hline Screening characteristics & Subclass & AF (\%) & No AF (\%) & OR $(95 \% \mathrm{Cl})$ & $P$ value \\
\hline \multirow[t]{2}{*}{ Sex } & Male & 31 (18.3) & $138(81.7)$ & 1.000 & \\
\hline & Female & $39(16.7)$ & 195 (83.3) & 0.890 (0.530 to 1.497$)$ & 0.661 \\
\hline Age & Mean \pm SD & $66.4 \pm 19.0$ & $58.8 \pm 18.5$ & 1.025 (1.009 to 1.041$)$ & 0.002 \\
\hline \multirow[t]{2}{*}{ Education } & Informal & $27(24.8)$ & $82(75.2)$ & 1.000 & \\
\hline & Formal & $43(14.6)$ & $251(85.4)$ & 0.520 (0.303 to 0.895$)$ & 0.018 \\
\hline \multirow[t]{2}{*}{ Income } & Low & $34(22.1)$ & $120(77.9)$ & 1.000 & \\
\hline & Medium/high & $36(14.5)$ & $213(85.5)$ & 0.597 (0.355 to 1.003$)$ & 0.051 \\
\hline \multirow[t]{2}{*}{ Residence } & Urban & $26(15.4)$ & $143(84.6)$ & 1.000 & \\
\hline & Rural & $44(18.8)$ & $190(81.2)$ & 1.274 (0.749 to 2.166$)$ & 0.372 \\
\hline \multirow[t]{2}{*}{ Health insurance } & Yes & $31(15.4)$ & $171(84.7)$ & 1.000 & \\
\hline & No & 39 (19.4) & $162(80.6)$ & 0.753 (0.448 to 1.264$)$ & 0.283 \\
\hline \multirow[t]{4}{*}{$\mathrm{BMI}\left(\mathrm{kg} / \mathrm{m}^{2}\right)$} & Underweight & $4(15.4)$ & $22(84.6)$ & 1.000 & \\
\hline & Normal & $43(22.5)$ & $148(77.5)$ & 1.598 (0.522 to 4.889$)$ & 0.411 \\
\hline & Overweight & $15(12.5)$ & $105(87.5)$ & 0.786 (0.238 to 2.595$)$ & 0.692 \\
\hline & Obese & $8(12.1)$ & $58(87.9)$ & 0.759 (0.207 to 2.774$)$ & 0.676 \\
\hline $\begin{array}{l}\text { Duration of heart failure } \\
\text { (years), median (IQR) }\end{array}$ & & $6.5(3-13)$ & $4(2-7)$ & 1.076 (1.034 to 1.076$)$ & $<0.001$ \\
\hline \multirow[t]{2}{*}{ NYHA function class } & I/II & $9(10.8)$ & 74 (89.2) & 1.000 & \\
\hline & III/IV & $61(19.1)$ & $259(80.9)$ & 1.937 (0.918 to 4.083$)$ & 0.083 \\
\hline \multirow[t]{2}{*}{ Diabetes mellitus } & No & $55(18.0)$ & $251(82.0)$ & 1.000 & \\
\hline & Yes & $15(15.5)$ & $82(84.5)$ & 0.835 (0.448 to 1.556$)$ & 0.57 \\
\hline \multirow[t]{2}{*}{ Alcohol } & No & 26 (12.2) & $188(87.8)$ & 1.000 & \\
\hline & Yes & $44(23.3)$ & $145(76.7)$ & 2.194 (1.290 to 3.732 ) & 0.004 \\
\hline \multirow[t]{2}{*}{ Cigarette smoking } & No & $53(16.3)$ & $273(83.7)$ & 1.000 & \\
\hline & Yes & $17(22.1)$ & $60(77.9)$ & 1.459 (0.790 to 2.696$)$ & 0.227 \\
\hline
\end{tabular}

AF, atrial fibrillation; BMI, body mass index; NYHA, New York Heart Association.

diagnosed with advanced HF (III/IV NYHA class). The most predominant comorbidity was hypertension, with 323 cases $(80.2 \%)$. Ninety-seven $(97,24.1 \%)$ had concurrent diabetes mellitus. Nearly half of the participants $(189,46.9 \%)$ reported a social history positive for alcohol consumption, and 77 (19.1\%) had a smoking history.

\section{Prevalence of AF}

Of the 403 study participants with heart failure, 70 (17.4\%) participants had AF detected on screening ECG. Of these, 29 out of $70(41.4 \%)$ had previously been diagnosed with AF and 41 out of 70 (58.6\%) were new diagnoses. Twenty-five per cent (6/24) of participants with a history Rheumatic Heart Disease (RHD) had atrial fibrillation (table 1).

\section{Sociodemographic correlates of AF}

In a univariable logistic regression model (table 2), advanced age, low income, informal education, alcohol consumption and longer HF duration were significantly associated with AF. In the multivariable model (table 3), lower income (high income adjusted OR (aOR) 0.5,
95\% CI 0.3 to 0.9 ), duration of $\mathrm{HF}$ (aOR $1.05,95 \%$ CI 1.0 to 1.1) and alcohol consumption (aOR 2.1, 95\% CI 1.2 to 3.8) were associated with AF.

\section{Clinical and physical exam correlates of AF}

By univariate logistic regression (table 4), irregular pulse rhythm, higher baseline heart rate and greater

Table 3 Multivariate logistic regression of demographic factors associated with atrial fibrillation

\begin{tabular}{|c|c|c|}
\hline Variable & aOR (95\% Cl) & $P$ value \\
\hline Age & 1.006 (0.987 to 1.025$)$ & 0.564 \\
\hline Education (formal) & 0.659 (0.354 to 1.223$)$ & 0.186 \\
\hline Income (med/high) & 0.531 (0.306 to 0.920$)$ & 0.024 \\
\hline $\begin{array}{l}\text { Duration of heart failure } \\
\text { (years) }\end{array}$ & 1.049 (1.000 to 1.100$)$ & 0.050 \\
\hline NYHA (III/IV) & 1.347 (0.612 to 2.962$)$ & 0.459 \\
\hline Alcohol consumption & $2.083(1.150$ to 3.771$)$ & 0.015 \\
\hline
\end{tabular}

aOR, adjusted OR; NYHA, New York Heart Association. 
Table 4 Univariate logistic regression for physical exam correlates of atrial fibrillation

\begin{tabular}{|c|c|c|c|c|c|}
\hline Screening characteristics & Subclass & AF (\%) & No AF (\%) & OR (95\% Cl) & P value \\
\hline Systolic blood pressure, median (IQR) & & $117(97-134)$ & $124(107-145)$ & 0.985 (0.974 to 0.996$)$ & 0.009 \\
\hline Diastolic blood pressure, median (IQR) & & $69(64-82)$ & $70(67-82)$ & $0.996(0.977$ to 1.016$)$ & 0.699 \\
\hline Pulse rhythm & Irregular & $63(61.2)$ & $40(38.8)$ & 65.925 (28.237 to 153.915$)$ & $<0.001$ \\
\hline Heart rate (bpm), median (IQR) & & $85(74-102)$ & $78(70-89)$ & $1.026(1.013$ to 1.040$)$ & $<0.001$ \\
\hline
\end{tabular}

AF, atrial fibrillation.

pulse deficit were linked to AF prevalence. Conversely, higher systolic blood pressure at baseline was associated with a decreased risk of having AF. With respect to physical examination findings, in the multivariate analysis (table 5), irregular pulse rhythm (OR 38.0, 95\% CI 15.3 to 94.4 ) and pulse deficit (OR 1.1, 95\% CI 1.0 to 1.2 ) are strongly suggestive of AF presence.

\section{Three-month mortality}

At the end of the 3-month follow-up, 120 (29.8\%) participants died, including $44.3 \%$ and $26.7 \%$ of those with and without $\mathrm{AF}$, respectively. Among the clinical variables (table 6), the factor most significantly associated with 3-month mortality was higher heart rate on ECG (HR 1.88, $95 \%$ CI 1.508 to 2.340$)$. Other noteworthy risk factors for death include $\mathrm{AF}$ (HR 1.75, 95\% CI 1.162 to 2.633), worse heart function (III/IV) on the NYHA scale (HR 1.64, 95\% CI 0.981 to 2.738 ), rural residence (HR $1.47,95 \%$ CI 1.006 to 2.150 ) and anaemia (HR $1.33,95 \%$ CI 1.012 to $1.738)$. Conversely, higher education, higher ejection fraction $(\geq 40 \%)$ and baseline systolic blood pressure within the normal range were associated with decreased HR. By multivariate analysis (table 7), increased ECG heart rate remained significantly associated with mortality. Collinearity was noted between $\mathrm{AF}$ and other measures of $\mathrm{HF}$, and the singular inclusion of AF displayed statistically significant mortality hazards when other diluting factors were omitted (table 8). On stratified analysis, death rate increased significantly with each increment in ECG heart rate, with a 3-month mortality of $21.5 \%$ for those with HR below $90 \mathrm{bpm}, 38.6 \%$ for those between 90 and 110

\begin{tabular}{lcc|}
\hline $\begin{array}{l}\text { Table } 5 \\
\text { factors associated with atrial fibrillation }\end{array}$ & \multicolumn{3}{l}{$\begin{array}{l}\text { Multivariate logistic regression for screening } \\
\text { Variable }\end{array}$} & OR $(95 \%$ Cl) & P value \\
\hline SBP & $0.987(0.967$ to 1.007$)$ & 0.186 \\
DBP & $1.019(0.988$ to 1.051$)$ & 0.240 \\
$\begin{array}{l}\text { Pulse rhythm } \\
\text { (irregular) }\end{array}$ & $38.001(15.292$ to 94.436$)$ & $<0.001$ \\
Heart rate & $1.003(0.984$ to 1.022$)$ & 0.778 \\
Pulse deficit & $1.110(1.018$ to 1.211$)$ & $\mathbf{0 . 0 1 8}$ \\
\hline
\end{tabular}

DBP, diastolic blood pressure; SBP, systolic blood pressure. bpm and $64.4 \%$ for patients with $>110 \mathrm{bpm}$ at baseline (figures 1 and 2). Additional analyses comparing the $\mathrm{AF}$ prevalence and 3-month mortality data for participants with echocardiograph confirmed HF (according to the ESC criteria) against those participants diagnosed based on clinical criteria alone was conducted. In the HFrEF cohort, death rate at 3 months was similar for those with $\mathrm{AF}$ and those without. For both HFpEF and clinical criteria diagnosis, there was a marked increase in the 3-month mortality in those with AF (table 9).

\section{DISCUSSION}

In this study, we sought to elucidate the prevalence and correlations of $\mathrm{AF}$, as well as the significant 3-month mortality risk factors for patients with HF in Tanzania. $\mathrm{AF}$ was common among our cohort: nearly one out of six $(17.4 \%)$ ambulatory adults had AF that was evident on a screening ECG. This high prevalence is similar to other reports from East Africa ${ }^{19}$ and is likely a result of poor post-diagnosis linkage to care. ${ }^{23}$ Of note, patients were more likely to be symptomatic if they were alcohol consumers, more elderly, or had longer HF duration. These are common risk factors for disruptions in cardiac electrophysiology, and in particular, heavy drinking is linked to sudden-onset supraventricular arrhythmias. ${ }^{24}$ Unlike age and HF duration, decreasing alcohol consumption is a lifestyle adjustment that patients can readily make to reduce their risk of developing AF. In addition, we found that socioeconomic factors associated with poverty, such as less education and lower monthly income, were correlated with AF. Previous studies ${ }^{19} 25$ cited these attributes as major barriers to outpatient care access, and potential contributors to poorer outcomes.

At the end of the 3-month follow-up, almost half of the patients with AF died (44.3\%). Participants with $\mathrm{HF}$ and concurrent $\mathrm{AF}$ experienced a $75 \%$ higher risk of dying in the first 3 months after enrolment compared with those with HF alone. This finding aligns with data from the Framingham Heart Study, which indicated a 1.5-fold to 1.9-fold increased mortality risk for patients with AF, further highlighting the need for early detection and treatment. ${ }^{26}$ Anaemia, a common condition in lowerincome countries, was significantly linked to mortality in 
Table 6 Univariate Cox hazard model with death as outcome

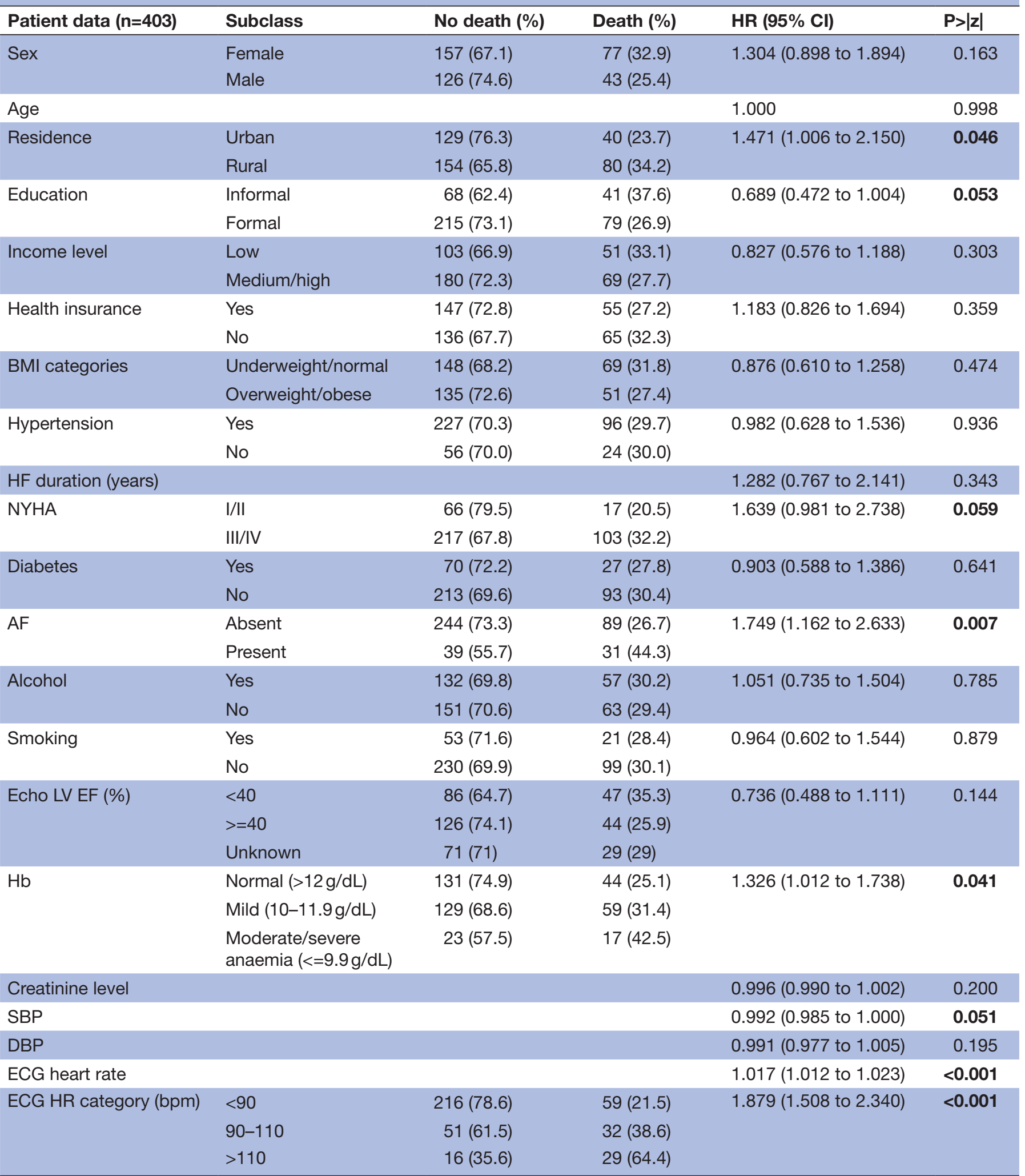

AF, atrial fibrillation; BMI, body mass index; DBP, diastolic blood pressure; $\mathrm{Hb}$, haemoglobin; HF, heart failure; LV EF, left ventricular ejection fraction; NYHA, New York Heart Association; SBP, systolic blood pressure.

our study participants, a finding corroborated by other reports from Tanzania. ${ }^{19}$ Lower systolic blood pressure was also associated with reduced survival, which was possibly a consequence of severely diminished left ventricular function. ${ }^{27}$ Finally, rural residence emerged as one of the significant predictors of mortality for outpatients with 


\begin{tabular}{llc}
\multicolumn{4}{l}{ Table 7} & Multivariate Cox HR \\
\hline Patient data & HR $(\mathbf{9 5 \%} \mathbf{~ C l})$ & P value \\
\hline Residence & $1.288(0.821$ to 2.021$)$ & 0.271 \\
\hline Education & $0.841(0.535$ to 1.322$)$ & 0.453 \\
Income level & $0.964(0.631$ to 1.471$)$ & 0.863 \\
\hline NYHA function class & $1.275(0.701$ to 2.318$)$ & 0.426 \\
AF & $1.030(0.629$ to 1.687$)$ & 0.907 \\
\hline Echo LV EF $(\%)$ & $0.910(0.578$ to 1.431$)$ & 0.682 \\
\hline ECG heart rate & $1.015(1.009$ to 1.021$)$ & $<0.001$ \\
\hline Haemoglobin & $1.062(0.760$ to 1.485$)$ & 0.723 \\
\hline SBP & $0.996(0.987$ to 1.005$)$ & 0.377 \\
\hline
\end{tabular}

AF, atrial fibrillation; LV EF, left ventricular ejection fraction; SBP, systolic blood pressure.

HF. In developing regions, wealthier populations often congregate in urban areas, leading to significant disparities in healthcare access and physician shortages in rural communities. ${ }^{28}$ These barriers contribute to delayed diagnosis of existing conditions as well as severely limited treatment options, thus further exacerbating the disease burden.

In both univariate and multivariate models of mortality, elevated heart rate on ECG was the strongest independent predictor of death within 3 months. ${ }^{29-31}$ Above the bounds of normal resting heart rate $(>110 \mathrm{bpm})$, an increase of 20 beats/min was associated with $>65 \%$ increased risk of death; a finding that remained significant even after adjusting for the presence of $\mathrm{AF}$ and other possible confounders. Furthermore, nearly $40 \%$ of people with ECG heart rates between 90 and 110 (ie, controlled by current guidelines) are dead at the end of the 3-month study period. It is likely that higher heart rate signals $\mathrm{HF}$ exacerbation. Our data identify a heart rate of $>125$ beats/min as extraordinarily high risk; therefore, this cut-off could help risk-stratify patients to appropriate care (ie, admission vs outpatient).

AF is specifically associated with higher mortality in the participants with confirmed HF with preserved ejection fraction as well as those with HF diagnosed based on clinical criteria alone. In fact, participants with $\mathrm{AF}$ in these

\begin{tabular}{lll}
$\begin{array}{l}\text { Table } 8 \\
\text { heart failure) }\end{array}$ & Multivariate Cox HR (without collinear measures of \\
\hline Patient data & HR $(95 \%$ Cl) & P value \\
\hline Residence & $1.343(0.912$ to 1.979$)$ & 0.136 \\
\hline Education & $0.813(0.551$ to 1.200$)$ & 0.297 \\
Income level & $0.931(0.645$ to 1.345$)$ & 0.704 \\
AF & $1.541(1.012$ to 2.345$)$ & $\mathbf{0 . 0 4 4}$ \\
Haemoglobin & $1.217(0.925$ to 1.602$)$ & 0.161 \\
SBP & $0.995(0.987$ to 1.003$)$ & 0.23 \\
\hline
\end{tabular}

$A F$, atrial fibrillation; SBP, systolic blood pressure.

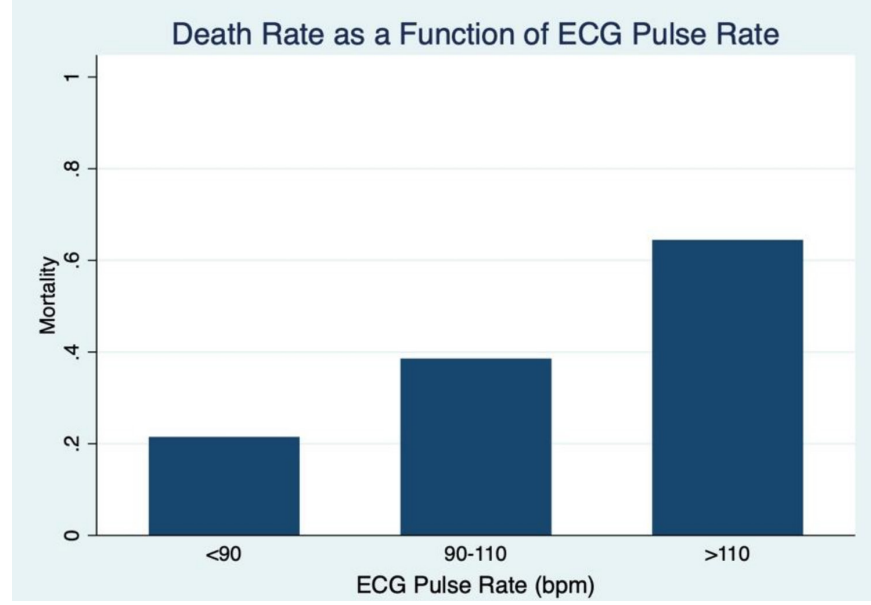

Figure 1 3-Month mortality per categorical heart rate.

two groups had higher mortality than those participants with confirmed HF with reduced ejection fraction. One possible explanation may be that those with worsened HF necessitate more physician visits. The greater contact with the healthcare system allows for more regular screenings, and any incidental findings to be noted and addressed in a timelier manner. Despite the growing global burden of AF, electrocardiograms are not routinely conducted in many HF clinics in low-income communities. ${ }^{9}$ Barriers to $\mathrm{AF}$ screening include the relative paucity of medical devices such as electrocardiograms, supplies such as ECG paper, and available specialty physicians per capita. ${ }^{12} 1321$ Encouragingly, our data imply that physical examination findings such as irregularly irregular pulse rate and pulse deficit are highly sensitive to detect patients with AF. Both measures can be ascertained with only palpation and a stethoscope and remain useful in clinical environments where ECG machines are not available.

There are limitations to this study. All participants were recruited from a single healthcare facility. Therefore, patients with HF included in this study may have different risk profiles than patients in other geographic locations

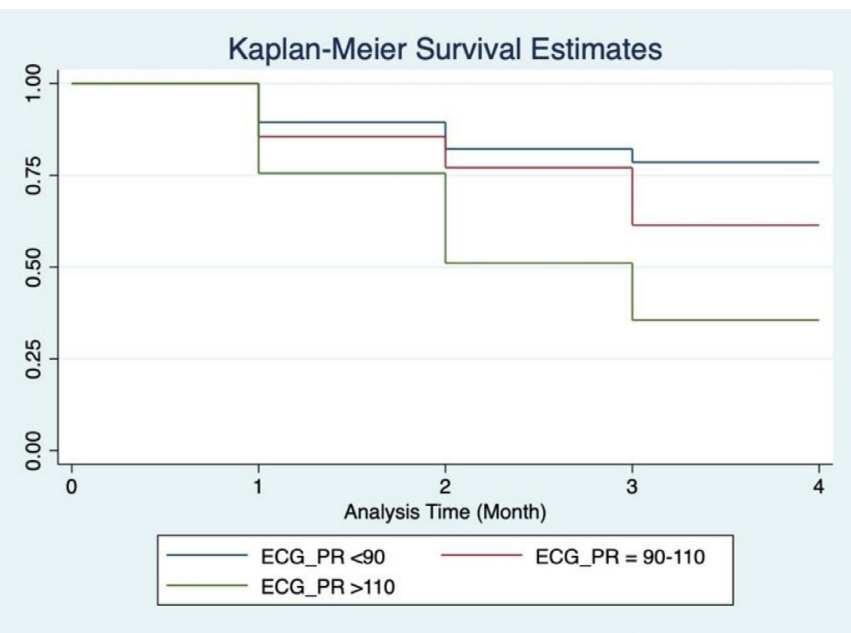

Figure 2 Kaplan-Meier curve for 3-month survival of adults with heart failure. 
Table 9 Stratified analysis of HFpEF, HFrEF, clinical criteria diagnosis and AF

\begin{tabular}{llrr}
\hline $\begin{array}{l}\text { Heart failure } \\
\text { condition }\end{array}$ & $\begin{array}{l}\text { AF/history } \\
\text { of AF }\end{array}$ & N & $\begin{array}{l}\text { 3-Month } \\
\text { mortality (\% of } \\
\text { subgroup N) }\end{array}$ \\
\hline $\begin{array}{l}\text { HFrEF-Echo } \\
\text { diagnosis }\end{array}$ & Yes & 35 & $12(34.3 \%)$ \\
HFpEF-Echo & Yes & 98 & $35(35.7 \%)$ \\
diagnosis & No & 143 & $13(48.1 \%)$ \\
Clinical criteria & Yes & 8 & $7(20.3 \%)$ \\
alone & No & 92 & $18(19.6 \%)$ \\
\hline
\end{tabular}

$A F$, atrial fibrillation; $\mathrm{HFpEF}$, heart failure with preserved ejection fraction ; HFrEF, heart failure with reduced ejection fraction.

and clinics. However, our study facility follows identical standards of care and the same protocols as other East African heart failure clinics, which promotes the generalisability of the results. Some aspects of the questionnaire, such as social history, rely on patient self-report, which may suffer from recall bias. Another study limitation is that we did not assess for rate-control medication adherence. This information could have helped differentiate deaths due to $\mathrm{AF}$ alone from those caused by poor drug adherence. While none of the subjects had a history of coronary artery disease, the diagnosis cannot be objectively ruled out from the existing clinical data. Additionally, because the focus of this study is the presence of $\mathrm{AF}$ and HF, the order in which the two conditions developed, and whether HF was due to non-ischaemic or ischaemic causes were not recorded.

\section{CONCLUSIONS}

Our data highlight the compounding morbidity and mortality of AF and HF in low-income and middle-income countries. AF is common, underdiagnosed and is associated with high mortality. In resource-limited settings, the presence of irregular heart rate and pulse deficit, along with affirmative responses to alcohol consumption and chronic HF, should push AF forward on the differential. Heightened resting heart rate should alert physicians to possible HF-related mortality. Common predictors that emerged for both $\mathrm{AF}$ and death are associated with systemic impediments to healthcare access and disparities in fiscal and human resource distribution. Thus, in order to effectively alleviate cardiovascular disease burden in Tanzania and other medically underserved regions, in general, there needs to be wider availability of preventative care and targeted screening of AF, particularly among vulnerable populations in rural communities. Our findings also provide a reminder to clinicians in low-income countries that physical examination still matters, and that HF patients with high heart rate deserve more careful clinical scrutiny.
Acknowledgements The study team is grateful for the support of administrators and healthcare providers at the Bugando Medical Center, and the participants who contributed to this research. We also wish to acknowledge the contributions of our data collection staff, Dr David Osima and Mr Evarist Msaki.

Contributors EA and YC: Study design, investigation, formal analysis and original draft preparation. EM: Investigation and review and editing. AM: Study design and review and editing. GAK and JRK: Review and editing. FK and RNP: Study design, supervision, and review and editing. All authors read and approved the final manuscript. EA is the Guarantor.

Funding This study was funded by a grant from the Mulago Foundation (Fund Number: N/A). RNP and JRK were both supported by grants from the National Institutes of Health (Fund Numbers: K01TW010281; K23 HL152926). The funding bodies had no role in the collection, analysis and interpretation of data and in writing the manuscript.

Competing interests None declared.

Patient and public involvement Patients and/or the public were not involved in the design, or conduct, or reporting or dissemination plans of this research.

Patient consent for publication Consent obtained directly from patient(s)

Ethics approval This study was approved by the CUHAS-BMC joint Ethics and Review Committee (CREC408/2019). All participants provided written informed consent before enrolment. Participants gave informed consent to participate in the study before taking part.

Provenance and peer review Not commissioned; externally peer reviewed.

Data availability statement Data are available upon reasonable request. The datasets and statistical code are available from the corresponding author on reasonable request.

Open access This is an open access article distributed in accordance with the Creative Commons Attribution Non Commercial (CC BY-NC 4.0) license, which permits others to distribute, remix, adapt, build upon this work non-commercially, and license their derivative works on different terms, provided the original work is properly cited, appropriate credit is given, any changes made indicated, and the use is non-commercial. See: http://creativecommons.org/licenses/by-nc/4.0/.

ORCID iD

Yunchan Chen http://orcid.org/0000-0002-0418-1705

\section{REFERENCES}

1 Keates AK, Mocumbi AO, Ntsekhe M, et al. Cardiovascular disease in Africa: epidemiological profile and challenges. Nat Rev Cardiol 2017:14:273-93.

2 Savarese G, Lund LH. Global public health burden of heart failure. Card Fail Rev 2017:3:7-11.

3 Hosseinpoor AR, Bergen N, Kunst A, et al. Socioeconomic inequalities in risk factors for non communicable diseases in lowincome and middle-income countries: results from the world health survey. BMC Public Health 2012;12:912.

4 Mensah GA, Roth GA, Sampson UKA, et al. Mortality from cardiovascular diseases in sub-Saharan Africa, 1990-2013: a systematic analysis of data from the global burden of disease study 2013. Cardiovasc J Afr 2015;26:S6-10.

5 Agbor VN, Essouma M, Ntusi NAB, et al. Heart failure in sub-Saharan Africa: a contemporaneous systematic review and meta-analysis. Int J Cardiol 2018;257:207-15.

6 Kingery JR, Yango M, Wajanga B. Heart failure, post-hospital mortality and renal function in Tanzania: a prospective cohort study. Int J Cardiol 2017;243:311-7.

7 Naser N, Dilic M, Durak A, et al. The impact of risk factors and comorbidities on the incidence of atrial fibrillation. Mater Sociomed 2017;29:231.

8 Stewart S, Wilkinson D, Hansen C, et al. Predominance of heart failure in the heart of Soweto study cohort: emerging challenges for urban African communities. Circulation 2008;118:2360-7.

9 Chugh SS, Roth GA, Gillum RF, et al. Global burden of atrial fibrillation in developed and developing nations. Glob Heart 2014;9:113.

10 Dries DL, Exner DV, Gersh BJ, et al. Atrial fibrillation is associated with an increased risk for mortality and heart failure progression in patients with asymptomatic and symptomatic left ventricular systolic dysfunction: a retrospective analysis of the SOLVD trials. studies of left ventricular dysfunction. J Am Coll Cardiol 1998;32:695-703. 
11 Maisel WH, Stevenson LW. Atrial fibrillation in heart failure: epidemiology, pathophysiology, and rationale for therapy. Am J Cardiol 2003;91:2-8

12 Peck R, Mghamba J, Vanobberghen F, et al. Preparedness of Tanzanian health facilities for outpatient primary care of hypertension and diabetes: a cross-sectional survey. Lancet Glob Health 2014;2:e285-92.

13 Katende D, Mutungi G, Baisley K, et al. Readiness of Ugandan health services for the management of outpatients with chronic diseases. Trop Med Int Health 2015;20:1385-95.

14 Ponikowski P, Voors AA, Anker SD. Esc guidelines for the diagnosis and treatment of acute and chronic heart failure. Eur Heart $J$ 2016;2016:2129-200.

15 McKee PA, Castelli WP, McNamara PM, et al. The natural history of congestive heart failure: the Framingham study. $N$ Engl J Med 1971;285:1441-6.

16 Gadzik J. "How much should I weigh?"--Quetelet's equation, upper weight limits, and BMI prime. Conn Med 2006;70:81-8.

17 Kirchhof P, Benussi S, Kotecha D, et al. 2016 ESC guidelines for the management of atrial fibrillation developed in collaboration with EACTS. Eur Heart J 2016;37:2893-962.

18 Sańchez J, Rosner SJ, B. Rosner, B.: fundamentals of biostatistics, third edition. PWS-Kent, Boston 1990, XV, 655 pp., us \$ 14.95, ISBN 0-534-91973-1. Biom. J. 1993;35:150.

19 Makubi A, Hage C, Lwakatare J, et al. Contemporary aetiology, clinical characteristics and prognosis of adults with heart failure observed in a tertiary hospital in Tanzania: the prospective Tanzania heart failure (TaHeF) study. Heart 2014;100:1235-41.

20 Agbor VN, Aminde LN, Tianyi F-L, et al. Atrial fibrillation among adults with heart failure in sub-Saharan Africa - prevalence, incidence and all-cause mortality: a systematic review and meta-analysis protocol. BMJ Open 2019;9:e022320.

21 Babyak MA. What you see may not be what you get: a brief, nontechnical introduction to overfitting in regression-type models. Psychosom Med 2004;66:411-21.
22 Bujang MA, Sa'at N, Sidik TMITAB, et al. Sample size guidelines for logistic regression from observational studies with large population: emphasis on the accuracy between statistics and parameters based on real life clinical data. Malays J Med Sci 2018;25:122-30

23 Familoni OB, Olunuga TO, Olufemi BW. A clinical study of pattern and factors affecting outcome in Nigerian patients with advanced heart failure. Cardiovasc J Afr 2007;18:308-11.

24 Djoussé L, Levy D, Benjamin EJ, et al. Long-Term alcohol consumption and the risk of atrial fibrillation in the Framingham study. Am J Cardiol 2004;93:710-3.

25 Maginga J, Guerrero M, Koh E, et al. Hypertension control and its correlates among adults attending a hypertension clinic in Tanzania. J Clin Hypertens 2016;18:207-16.

26 Benjamin EJ, Wolf PA, D'Agostino RB, et al. Impact of atrial fibrillation on the risk of death: the Framingham heart study. Circulation 1998;98:946-52.

27 Böhm M, Young R, Jhund PS, et al. Systolic blood pressure, cardiovascular outcomes and efficacy and safety of sacubitril/ valsartan (LCZ696) in patients with chronic heart failure and reduced ejection fraction: results from PARADIGM-HF. Eur Heart $J$ 2017;38:1132-43.

28 Leonard KL, Masatu MC. Variations in the quality of care accessible to rural communities in Tanzania. Health Aff 2007;26:w380-92.

29 Kannel WB, Kannel C, Paffenbarger RS, et al. Heart rate and cardiovascular mortality: the Framingham study. Am Heart $J$ 1987;113:1489-94.

30 Fox K, Ford I, Steg PG, et al. Heart rate as a prognostic risk factor in patients with coronary artery disease and left-ventricular systolic dysfunction (beautiful): a subgroup analysis of a randomised controlled trial. Lancet 2008;372:817-21.

31 Kapoor JR, Heidenreich PA. Heart rate predicts mortality in patients with heart failure and preserved systolic function. J Card Fail 2010;16:806-11. 